\title{
Variance Reduction and Signal-to-Noise Ratio: Reducing Uncertainty in Spectral Ratios
}

\author{
by Olga-Joan Ktenidou, Francisco J. Chávez-García, and Kyriazis D. Pitilakis
}

\begin{abstract}
This paper uses an unusually large dataset to study scatter in site-effect estimation, focusing on how the events that increase uncertainty can be removed from the dataset. Four hundred seventy-three weak motion earthquake records from the surface and bedrock of a 178-m-deep borehole in Aegion, Gulf of Corinth, Greece, are used to evaluate spectral ratios. A simple statistical tool, variance reduction (VR), is first used to identify two groups of events that lie closest and farthest from the average, which is considered here as the initial best estimate of the site response. The scatter in the original dataset is found to be due to the group of events with smallest VR. These events can be removed from the dataset in order to compute a more reliable site response. However, VR is not normally used to choose records for siteeffect studies, and it cannot be applied to the usual small datasets available. The signal-to-noise ratio (SNR) is normally used to this end, for which reason we investigate whether SNR can be used to achieve similar results as VR. Signal-to-noise ratio is estimated using different definitions. Data selection based on SNR is then compared to that using VR in order to define an SNR-based criterion that discriminates against events that, according to VR, increase scatter. We find that defining the SNR of a surface record as the mean value over a frequency range around the resonant peak (here, $0.5-1.5 \mathrm{~Hz}$ ) and using a cutoff value of 5 may be used in this case to exclude most events for which VR is small. This process is also applied to the downhole station, where we obtain similar results for a cutoff value of 3 .
\end{abstract}

\section{Introduction}

Site effects are often estimated using spectral ratios of earthquake data. The most usual are spectral ratios relative to a reference site, which are often called standard spectral ratios (SSR; e.g., Borcherdt, 1970; Chávez-García et al., 1990) and horizontal-to-vertical spectral ratios (HVSR), where horizontal component spectra are divided by the vertical component spectra recorded at the same site (e.g., Lermo and Chávez-García, 1993). If many records are available, higher quality data are often chosen based on signal-tonoise ratios. However, even when data quantity is satisfactory and data quality is acceptable, the scatter in the estimates of site amplification may be great. The reasons have been discussed previously (e.g., Jarpe et al., 1988; Chávez-García et al., 1990). If it were possible to detect the events that contribute most to our scatter and remove them from the dataset, then we would be able to decrease the uncertainty in siteeffect prediction.

The scatter of site-effect estimates is not often studied. Indeed, standard site-effect studies often use spectral ratios to

*Now at Institut de Radioprotection et de Sûreté Nucléaire (IRSN) DEI/SARG/BERSSIN, BP 17, 92262 Fontenay-aux-Roses, Cedex, France. produce estimates of local amplification based on a dozen or less recorded events. Small datasets may make it problematic to even define an average transfer function. In this paper we use an exceptionally large dataset consisting of 473 small earthquakes recorded by the vertical array CORSSA (Corinth Soft Soil Array) installed near the city of Aegion, in the Gulf of Corinth, Greece. This large dataset allows us to investigate the scatter of spectral ratios. We examine whether certain events in our dataset contribute more than their share to the scatter about the average value and how these can be found and removed using two methods, the signal-to-noise ratio (SNR) and variance reduction. Variance reduction (VR) is a simple statistical tool that evaluates goodness-of-fit between observations and predictions of a model. It has often been used in the determination of fault plane solutions. In this paper, thanks to the large number of events, we can use VR to estimate the agreement between an individual spectral ratio and the average spectral ratio for the complete dataset, considered as the initial best estimate. We can use VR to classify individual events in terms of how near or how far their ratios lie from the average. Those events lying farthest from the average can then be rejected. 
Variance reduction, however, cannot be used to screen small datasets. In these cases, data quality is usually assessed using SNR. When we select events based on SNR, we choose whether to include the spectral ratio of a particular event in the computation of the average based on whether a criterion regarding the ratio between signal and noise is met. Varying SNR criteria are used here, and we search for the SNR criterion that best reproduces the record selection obtained using VR. Thus, we are able to propose an SNR selection criterion that best matches the selection made through VR and that may be used to choose events in the smaller datasets usually available to estimate site effects.

\section{Signal-to-Noise Ratio and Variance Reduction}

Signal-to-noise ratio is often used in site response studies to choose higher quality data from the available dataset. According to Borcherdt (1970), SSR in the frequency band where SNR is high should isolate local site effects. Signal-tonoise ratio is usually defined in this context as the ratio of the Fourier spectrum of the signal time window by the spectrum of the pre-event noise. The length of these windows can vary greatly. Both acceleration and velocity data yield similar SNR (Jarpe et al., 1988). Ratios for the two horizontal components are usually computed independently, producing comparable (Borcherdt and Gibbs, 1976; Kato et al., 1995; Lachet et al., 1996) or dissimilar results (Wong et al., 1977). Signal-tonoise ratio depends on frequency and decreases rapidly beneath $0.4-1 \mathrm{~Hz}$ for small earthquakes (Malagnini et al., 1996; Steidl et al., 1996).

When computing spectral ratios, SNR is usually evaluated in the frequency domain, its spectral amplitude values are compared with a chosen cutoff threshold, and data whose SNR fall below this value are removed. The lowest SNR cutoff value used is 2 (e.g., Borcherdt and Gibbs, 1976; Rogers et al., 1984; Darragh and Shakal, 1991; Satoh et al., 2001; Lozano et al., 2009). Most studies use the value of 3 (e.g., Field and Jacob, 1995; Theodulidis et al., 1996; Lachet et al., 1996; Bonilla et al., 1997, 2002; Di Giacomo et al., 2005; Sawazaki et al., 2009). Riepl et al. (1998) report that their results are no different for thresholds of 3 or 5. Jarpe et al. (1988), Cramer (1995), and Thompson et al. (2009) use a cutoff value of 5, while Steidl et al. (1996) and Malagnini et al. (1996) use a value of 10.

Contrary to SNR, variance reduction (VR) is not usually applied in site-effect studies, but rather in source studies (e.g., Chi et al., 2001; Skarlatoudis et al., 2003; Scherbaum et al., 2004). Variance reduction may also be used to discriminate between higher and lower quality data. Variance reduction is defined in different ways according to different authors. In this paper, we define VR as

$$
\mathrm{VR}=1-\sum\left(\frac{\text { discrepancy }}{\text { observation }}\right)^{2} \text {, }
$$

where the term discrepancy means the difference between an observation and an expected value based on a model.
As defined in equation (1), VR assumes values within the range $(-\infty, 1]$. It equals 1 when there is a perfect match between expected and observed values, while as values become smaller they indicate poorer fit. Equation (1) resembles the chi-square fit test, but the normalization of the residuals is made with respect to the data amplitude rather than to the standard deviation. Evidently, if we do not know the local amplification, it is not possible to evaluate VR. We do not have a model that we can trust completely to predict site amplification at Aegion. However, the sheer size of our dataset makes for a very robust observed average amplification. It is this average transfer function that will allow us to compute VR.

\section{Data and Spectral Ratios}

Our dataset comes from the Corinth Soft Soil Array (CORSSA; see the Data and Resources section), which is installed near the city of Aegion, in the southern part of the Gulf of Corinth, Greece, one of the most active seismic areas in Europe (Fig. 1). Aegion is crossed by a fault with an escarpment of roughly $80 \mathrm{~m}$ that, as shown in the cross-section, divides it in two levels. CORSSA vertical array is installed in the northern, lower part of the city, very near the coast. The soil profile at that point is known through various previous surveys (Pitilakis et al., 2004) to consist of soft, loose materials underlain by a stiff conglomerate $\left(V_{\mathrm{S}}>800 \mathrm{~m} / \mathrm{s}\right)$ at $155 \mathrm{~m}$ depth. The actual seismic bedrock is the limestone formation $\left(V_{\mathrm{S}}>1500 \mathrm{~m} / \mathrm{s}\right)$, which lies at some $700 \mathrm{~m}$, according to the cross-section of Figure 1 (Apostolidis et al., 2006). The array consists of four broadband 3D accelerometers at depths of $14,31,57$, and $178 \mathrm{~m}$, and one at the surface. The deepest accelerometer is located in the conglomerate formation; although this material is not as stiff as the limestone, it has been shown (Ktenidou, 2010) that the station can be used as reference (the average HVSR value at depth for events used in the present study is near unity in the $0.1-10 \mathrm{~Hz}$ range).

The dataset used consists of 473 earthquakes recorded over a period of 6 years since the array began to operate in 2002. Figure 2 shows the epicentral distribution. This dataset has been used previously to evaluate site effects (Ktenidou, 2010), showing the large importance of the geometry of the sediments relative to the Aigion fault. For this reason, the horizontal components studied here are rotated to the radial and transverse components with respect to the fault escarpment. All data are weak motion, and only records from the surface and deepest stations are analyzed here.

The data were processed as follows. Time-histories were synchronized, baseline-corrected, and $10 \%$ cosine-tapered at the edges. They were band-pass-filtered between 0.2 and $15 \mathrm{~Hz}$ using a zero-phase Butterworth filter with four poles. Then they were rotated to the radial and transverse directions relative to the Aigion fault. Fourier spectra were calculated and smoothed four times with a Hanning window and spectral ratios were computed. The spectral ratios were interpolated 

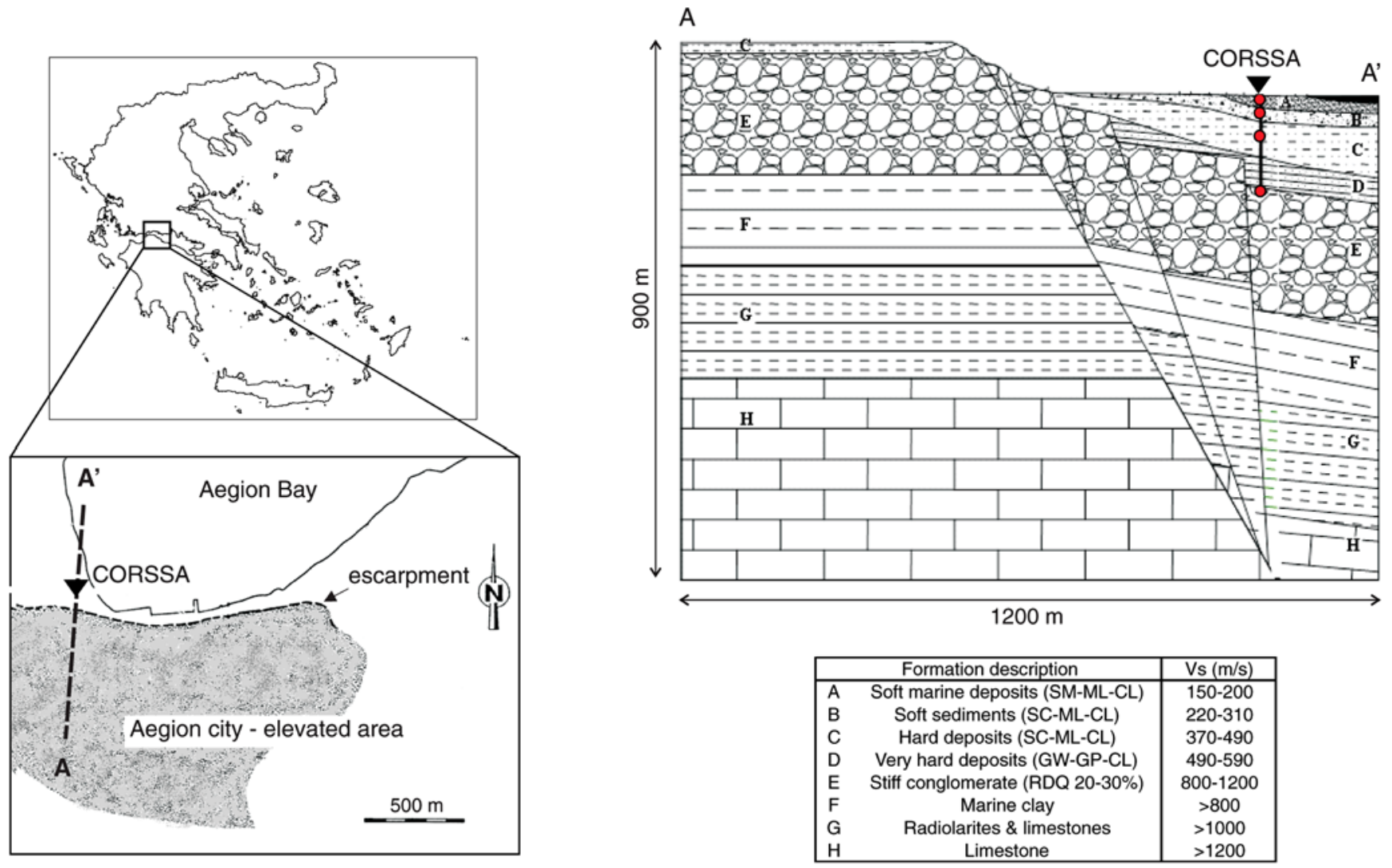

Figure 1. Left diagram: location of the city of Aegion, Gulf of Corinth, Greece (adapted from Athanasopoulos et al., 1999). The Corinth Soft Soil Array and the Aegion fault escarpment dividing the city in two levels are marked along with cross-section A-A'. Top right diagram: cross-section A-A', perpendicular to the slope and to the basin formed by the sediments beneath the Gulf (after Apostolidis et al., 2006). The bottom right table gives the $V_{\mathrm{S}}$ for each layer in the cross-section (after Apostolidis et al., 2006). The color version of this figure is available only in the electronic edition.

to a common frequency step for averaging purposes. The average and standard deviation were calculated using the decimal logarithms of the spectral ratio values, assuming that the data follow a normal distribution at all frequencies (this common assumption is investigated in the following section). We also computed the $95 \%$ confidence limits of the average value based on a two-tailed $t$-student distribution. Spectral ratios were computed using complete records (with durations between 41 and 180 s) rather than just an $S$ window. Theodulidis et al. (1996) argue that entire time-histories recorded are better to compute spectral ratios because it is not straightforward to separate wave types due to scattering effects in the signal following $P$-wave arrival. In the case of our dataset, Ktenidou (2010) found that the results using the complete records and using smaller body-wave windows are similar.

We computed HVSR for all surface records and SSR between all surface and 178-m-depth record pairs. The result is shown in Figure 3, where the 95\% confidence intervals of the average values are plotted for the three components and standard deviation is shown separately. Both SSR and HVSR show the fundamental frequency of the site to be $0.9 \mathrm{~Hz}$. This has also been estimated through noise measurements and 1D

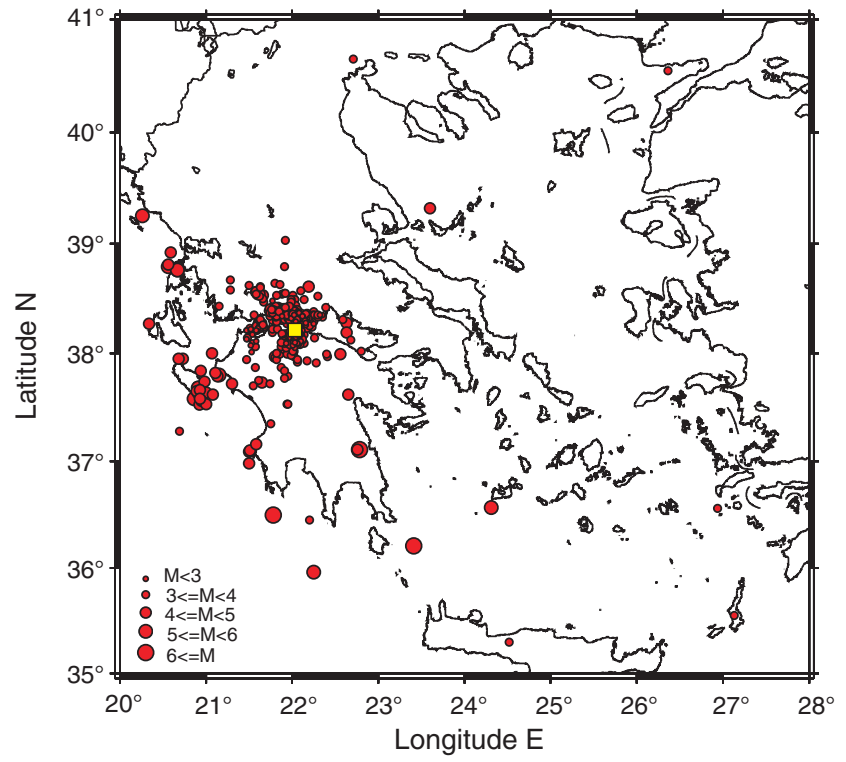

Figure 2. Epicenter distribution for the 473 events of the dataset. The size of the circle scales with moment magnitude (dark circles). CORSSA site is marked by a square. The color version of this figure is available only in the electronic edition. 

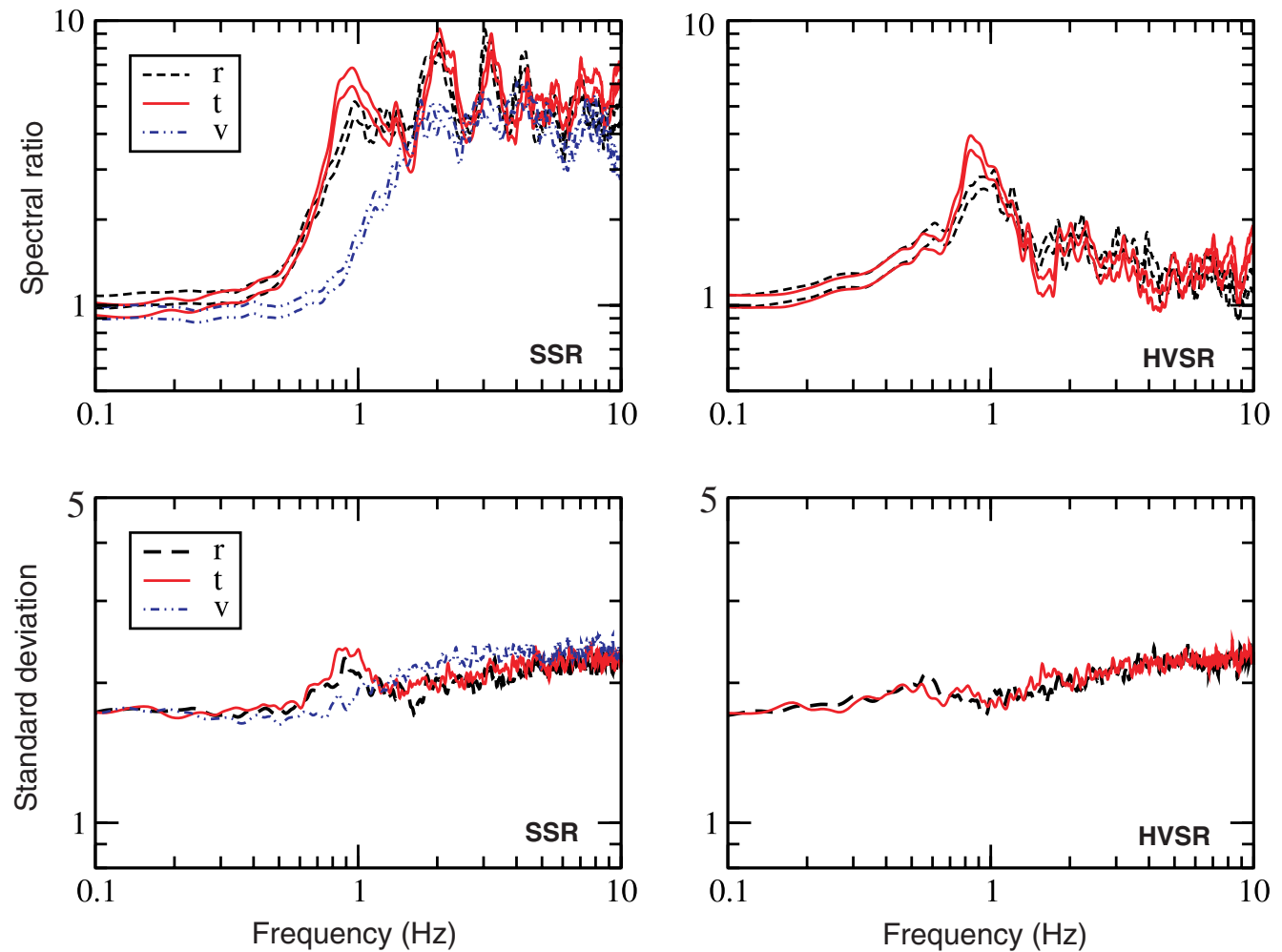

Figure 3. Horizontal-to-vertical spectral ratios (HVSR) at the surface and standard spectral ratios at the surface with respect to the downhole station at $178 \mathrm{~m}$ (SSR) for the complete dataset (473 events), for the radial ( $r$ ), transverse $(t)$ and vertical $(v)$ components. Top: $95 \%$ confidence limits of the logarithmic mean spectral ratio values. Bottom: standard deviation of the mean. Scatter is larger for the SSR technique around the fundamental peak $(0.9 \mathrm{~Hz})$. The color version of this figure is available only in the electronic edition.

theoretical analysis by Apostolidis et al. (2006), as well as through numerical analysis by Ktenidou (2010). In the frequency band of resonance, from 0.5 to $1.5 \mathrm{~Hz}$, the scatter for SSR is larger than that for HVSR (a common observation, e.g., Lermo and Chávez-García, 1993; Bard, 1998), with a maximum value of 2.4 as opposed to 2.0 , respectively. For frequencies outside this range, the standard deviation is similar for both ratios. Figure 3 shows that the vertical component is amplified between the bedrock and the surface. Though this amplification is most significant for frequencies higher than $1.5-2 \mathrm{~Hz}$, it is also visible near the fundamental frequency. This amplification partly explains the differences between the amplitudes of SSR and HVSR, which have been observed before (Field and Jacob, 1995; Theodulidis et al., 1996).

We have used the station at depth as a reference for SSR because no surface station on rock outcrop near our site is available. Steidl et al. (1996) and Şafak (1997) caution that care be taken when using buried instruments as a reference, because the downgoing wave field may cause destructive interference and hence produce pseudoresonances in the spectral amplification estimates. For this reason, we have checked the suitability of the downhole station as a reference site. Figure 4 shows the $95 \%$ confidence intervals of the average HVSR computed at depth and the corresponding standard deviation. The result was very similar for the two hori- zontal components and its amplitude was about unity, with fluctuations of the confidence interval from 0.7 to 1.5 between 0.1 and $10 \mathrm{~Hz}$. We verified that these small fluctuations came from interference of downward propagating waves through comparison with numerical modeling (Ktenidou, 2010). However, interference of downward propagating waves affected mainly frequencies larger than $1.5 \mathrm{~Hz}$, for which the scatter also increased. Thus, the uncertainty introduced by this effect did not affect our conclusions regarding the fundamental peak. We concluded that the station at $178 \mathrm{~m}$ depth could be used as a reference site for the SSR technique.

\section{Distribution Function}

The large size of our dataset allows us to explore the validity of the normal distribution of the spectral ratio logarithm, which we have assumed to compute spectral ratio averages and which is usually assumed. The validity of the assumption is investigated for both horizontal components of the SSR and HVSR calculated at the surface (i.e., four cases) at frequency values: $0.5,1,1.5,2,3,5,7$, and $9 \mathrm{~Hz}$. For each case and frequency, we use 24 bins of 0.12 width to sort the logarithms of the spectral values. The number of events in each bin is normalized by the total number of events and by the bin width. The results are shown in Figure 5a for the radial component of HVSR. The histograms shown in this 

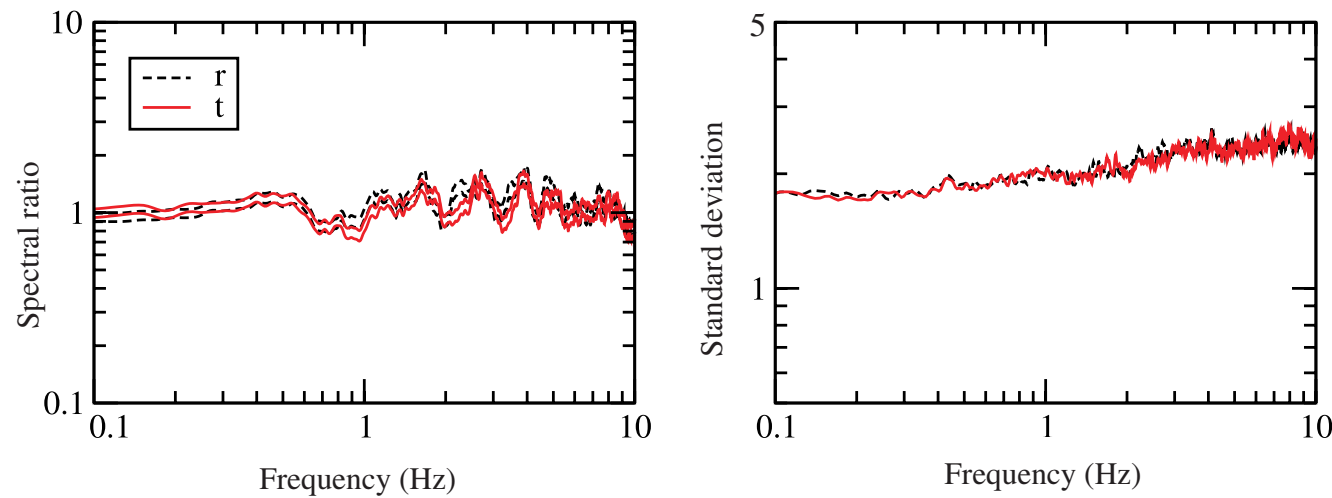

Figure 4. Horizontal-to-vertical spectral ratios (HVSR) at the downhole station at $178 \mathrm{~m}$ for the entire dataset (473 events), for radial ( $r$ ) and transverse $(t)$ component. Left: 95\% confidence limits of the logarithmic mean values. Right: standard deviation of the mean. The station at depth is considered to be an adequate reference station. The color version of this figure is available only in the electronic edition.

figure resemble closely the normal probability density function plotted onto them, computed from the respective average and standard deviation values (Wessa, 2009). Figure 5b shows histograms of cumulative frequency normalized by the total number of events, which closely resemble the theoretical cumulative frequency function calculated for the data assuming normal distribution of the log.

We use three different goodness-of-fit tests to assess whether the normal distribution is indeed a good fit for the distribution of the log of the spectral ratios computed. We use the Kolmogorov-Smirnov, Anderson-Darling, and chisquare statistical tests. They are used by Restrepo-Vélez and Bommer (2003) to explore the distribution of PGA residuals of ground-motion prediction equations and are described in NIST (2001). For each of the four spectral ratio cases and each of the eight frequency values, we compare the statistic calculated by the data array with the critical values according to the three tests for given significance levels $(a=1 \%, 2 \%, 5 \%, 10 \%$, and 20\%). If the value calculated is higher than the critical value of a specific test, then the normal distribution is rejected by the test for that level. The results are shown in Table 1, in which the cases where the assumption was rejected are marked by X. We conclude that the normal probability density function is accepted by all tests for the log of HVSR at all frequencies checked. For the log of SSR, it is rejected by the chi-square test for all significance levels and by the other two tests for $a=10 \%$ and $20 \%$ at $0.5 \mathrm{~Hz}$ and $1 \mathrm{~Hz}$ and accepted at the other frequencies. At the resonant frequency, the normal distribution is not the best one to describe the log of SSR. Figure 6 shows, however, that the histograms for all eight frequencies point to a unimodal distribution that for most frequencies is very near the normal one, suggesting that the initial assumption is valid.

\section{Results Using Variance Reduction}

We present now the results using variance reduction to identify events that contribute to the scatter already observed. We rewrite equation (1) as

$$
\mathrm{VR}=1-\sum_{i}\left(\frac{\mathrm{SR}\left(f_{i}\right)-\overline{\mathrm{SR}}\left(f_{i}\right)}{\mathrm{SR}\left(f_{i}\right)}\right)^{2}
$$

where $\operatorname{SR}\left(f_{i}\right)$ is the spectral ratio (SSR or HVSR) for a particular component at frequency $i$, and $\overline{\operatorname{SR}}\left(f_{i}\right)$ is the average of all spectral ratios at that frequency. The sum can be carried out over the entire frequency range or over a restricted range.

We consider the first spectral peak as the most important feature in SSR and HVSR. Also, Figure 3 shows that the scatter for SSR is significant around this peak. For these reasons, we evaluate VR only in the frequency range between 0.5 and $1.5 \mathrm{~Hz}$ (a range that is roughly symmetrical around $0.9 \mathrm{~Hz}$ on the log scale). We compute VR values for each horizontal component and for SSR and HVSR (four VR values per event) and sort events according to those values. We call good agreement between an individual ratio and the average when at least three of its four VR values are within the 20\% largest values. The corresponding events are called VR+ events. We call bad agreement ratios those with at least three out of four VR values within the $20 \%$ smaller values. The corresponding events are called VR- events. Our results show that our dataset includes $55 \mathrm{VR}+$ events (with values above 0.7) and $47 \mathrm{VR}$ - events (with values below zero). We note that we do not define VR as a percentage because of these negative values.

Figure 7 shows the 95\% confidence intervals of the mean for SSR and HVSR for all events in our dataset and for the two groups of events, VR- and VR+. The SSR for VRevents fail to identify the resonant peak, while the VR+ events show it clearly. The results for HVSR show that the fundamental peak appears clearly for the VR- events, though its amplitude is smaller than for the VR+ group. In all the plots in Figure 7, the 95\% confidence intervals corresponding to VR+ and VR- events are well separated from the corresponding intervals for the entire dataset.

Our results show that the average of all the events in the dataset is not necessarily an unbiased estimate of the transfer function. In the vicinity of the fundamental peak this average is biased toward lower values by the VR- events. A better 
(a)
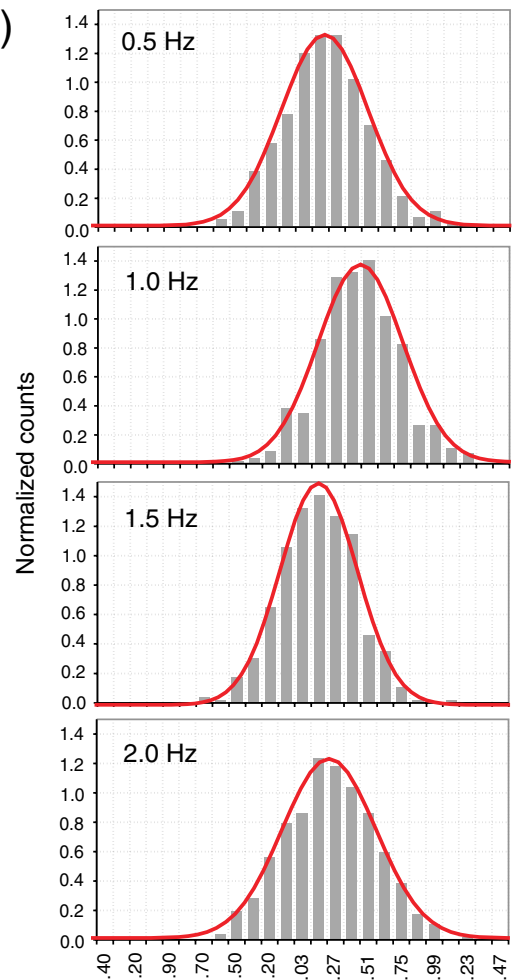

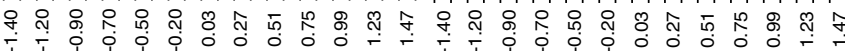
IogHVSR

(b)

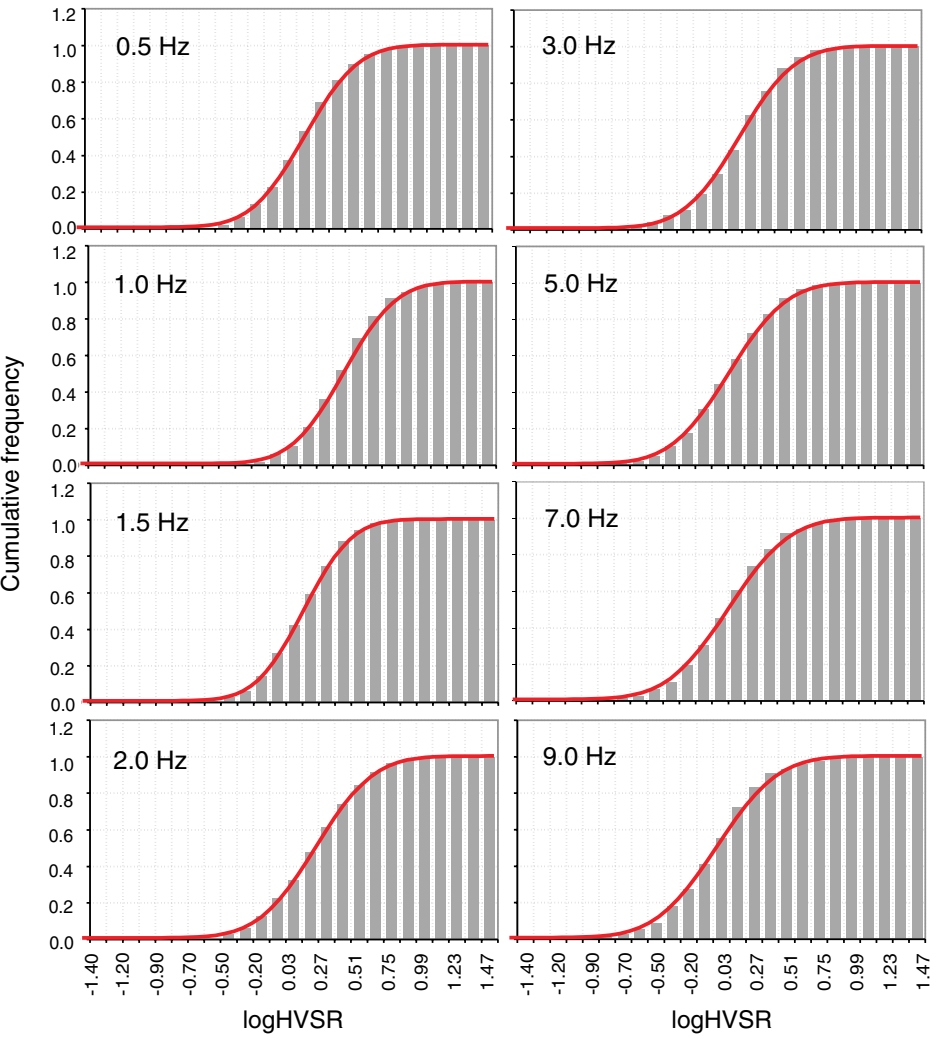

Figure 5. (a) Histograms of normalized counts for spectral values at 8 frequency values $(0.5,1,1.5,2,3,5,7$, and $9 \mathrm{~Hz})$. Horizontal axis shows bins for the log value of the radial component of HVSR. Vertical axis shows occurrences within each bin normalized by the total number of events (473) and the bin width (0.12). The normal distributions fitted to the data are plotted as solid lines. (b) Histograms of cumulative frequency for the same data. Horizontal axis shows bins for the log value of the radial component of HVSR. Vertical axis shows cumulative frequency normalized by the total number of events (473). The normal distribution cumulative probability curves fitted to the data are shown as solid lines. The color version of this figure is available only in the electronic edition. 
Table 1

Use of Three Statistical Tests to Evaluate the Assumption of Normal Distribution for the Log of SSR and HVSR*

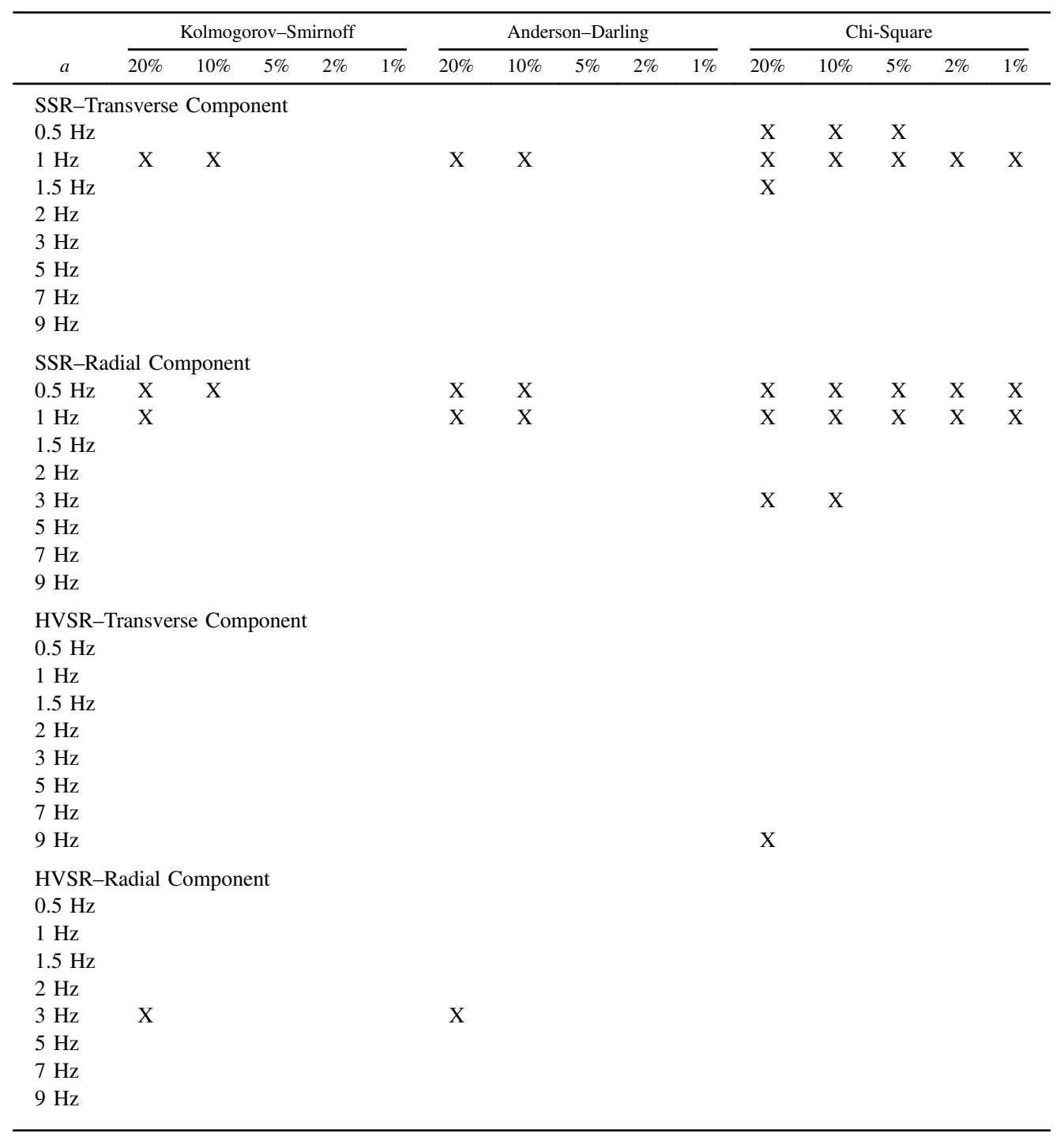

*An $\mathrm{X}$ indicates that the assumption was rejected for the specific frequency and significance level.

estimate is obtained when we use only events with small scatter, that is, VR+ events. Of course, deciding which events show a small scatter must by force be based on the average computed for the complete dataset, and only has meaning when the dataset is as large as the one at hand. The unimodal, normal distribution we found previously justifies the treatment of VR- events as outliers that lower the average transfer function. Hence, the first estimate using the complete dataset can be refined by removing them.

Our definition of VR normalizes the distance between each observation and the average with the respective observation amplitude. This normalization introduces a bias against very small amplitudes, allowing us to remove the events that miss the fundamental peak. Variance reduction is not used here as an objective goodness-of-fit index, since the VR+ group does not identify exactly the overall average transfer function, but selects higher values to compensate for the average transfer function being biased toward lower values.

The standard deviation of the mean for the spectral ratios of the complete dataset and the two groups of events is shown in Figure 8. The uncertainty of the VR+ events is smaller than that of the VR- events for the horizontal components in the frequency range of interest $(0.5-1.5 \mathrm{~Hz})$, as expected. It is usually assumed that standard deviation values of spectral ratios are within a factor of 2 (Tucker and King, 1984; Jarpe et al., 1988; Chávez-García et al., 1990; Bard, 1998). Our results show that, for SSR, the average standard deviation of the entire dataset around the fundamental frequency is 2.4. This is almost equal to the standard deviation of the VR- events. For the VR+ events, average standard deviation of SSR is between 1.5 and 1.8. Thus, the larger scatter of the 
(a)
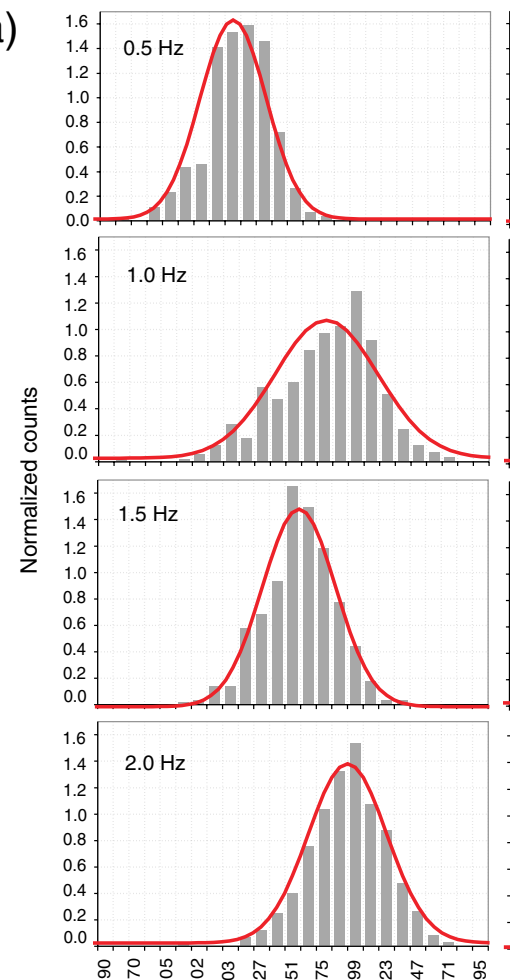

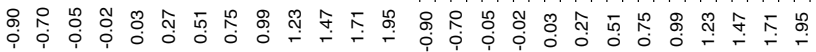

logSSR

(b)

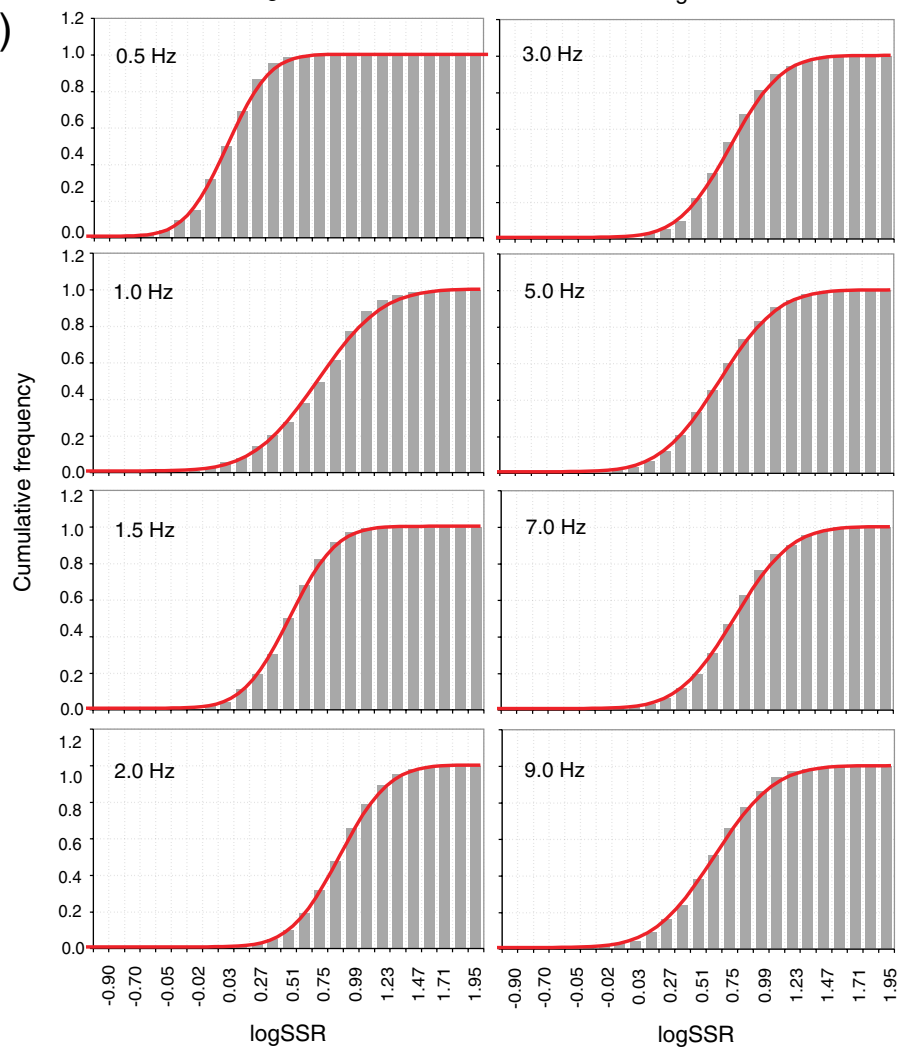

Figure 6. (a) Histograms of normalized counts for spectral values at 8 frequency values $(0.5,1,1.5,2,3,5,7$, and $9 \mathrm{~Hz})$. Horizontal axis shows bins for the log value of the transverse component of SSR. Vertical axis shows occurrences within each bin normalized by the total number of events (473) and the bin width (0.12). The normal distributions fitted to the data are plotted as solid lines. (b) Histograms of cumulative frequency for the same data. Horizontal axis shows bins for the log value of the $r$ component of SSR. Vertical axis shows cumulative frequency normalized by the total number of events (473). The normal distribution cumulative probability curves fitted to the data are plotted as solid lines. The color version of this figure is available only in the electronic edition. 

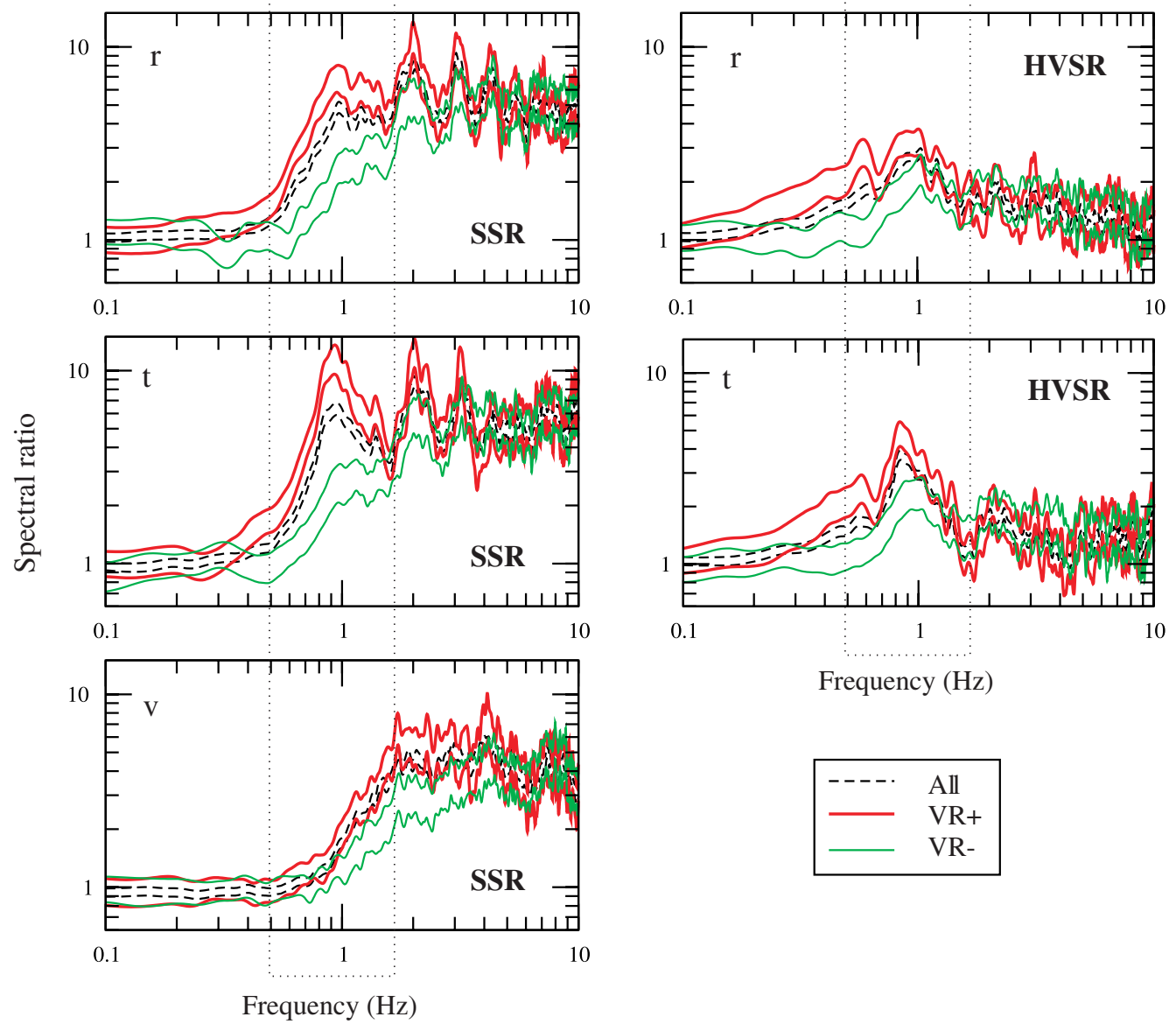

Figure 7. Confidence limits of $95 \%$ of the mean values of SSR and HVSR for the radial $(r)$, transverse $(t)$, and vertical $(v)$ components. Results are shown for the entire dataset (dashed lines) and for the VR - and VR+ events (thin and thick solid lines, respectively). The dotted boxes mark the frequency range $0.5-1.5 \mathrm{~Hz}$. The VR- group systematically fails to capture the SSR fundamental peak. The color version of this figure is available only in the electronic edition.

complete dataset comes from events in the VR- group. For HVSR, average standard deviation of the entire dataset lies between those of the two groups, VR+ and VR-.

We checked whether the events that contribute most to the scatter (VR- events) can be identified based on their parameters: epicentral distance to CORSSA, source depth, magnitude, or azimuthal distribution relative to CORSSA. The results are negative. Figure 9, for example, compares the distribution of magnitude and focal depth with epicentral distance for VR- events versus the entire dataset. Figure 10 compares the epicentral distribution for VR- events with that for the entire dataset. These figures show that VRevents have moment magnitudes, epicentral distances, and focal depths that cannot be differentiated from those for the complete dataset. Variance reduction is the only way to identify these events.

\section{Signal-to-Noise Ratios}

A selection of earthquake records for site-effect studies based on VR is not usually possible because the number of available records is typically small. Signal-to-noise ratio (SNR) is more frequently used to select high quality records. In this section, we estimate SNR for our dataset using different definitions and compare the results with those of the previous section.

Signal-to-noise ratio is defined as the ratio between the Fourier amplitude spectrum of the $S$-wave window and that of the pre-event noise. The spectrum of noise is computed using the first $8 \mathrm{~s}$ of each record, which correspond to the signal recorded before the trigger (the stations have a preevent memory of $10 \mathrm{~s}$ ). For this reason, we cannot use the entire accelerograms as signal windows when computing SNR, although we use them to compute the spectral ratios. The signal window chosen to compute SNR is taken as the 8-s window starting immediately after the trigger. Each window is $10 \%$ tapered at the edges before Fourier spectra are computed. For our dataset, SNR is smallest below $1 \mathrm{~Hz}$ and increases for frequencies larger than $2 \mathrm{~Hz}$.

We computed SNR for all events for two different frequency ranges: around the resonant peak $(0.5-1.5 \mathrm{~Hz})$ and over a wide frequency range $(0.1-10.0 \mathrm{~Hz})$. For each 

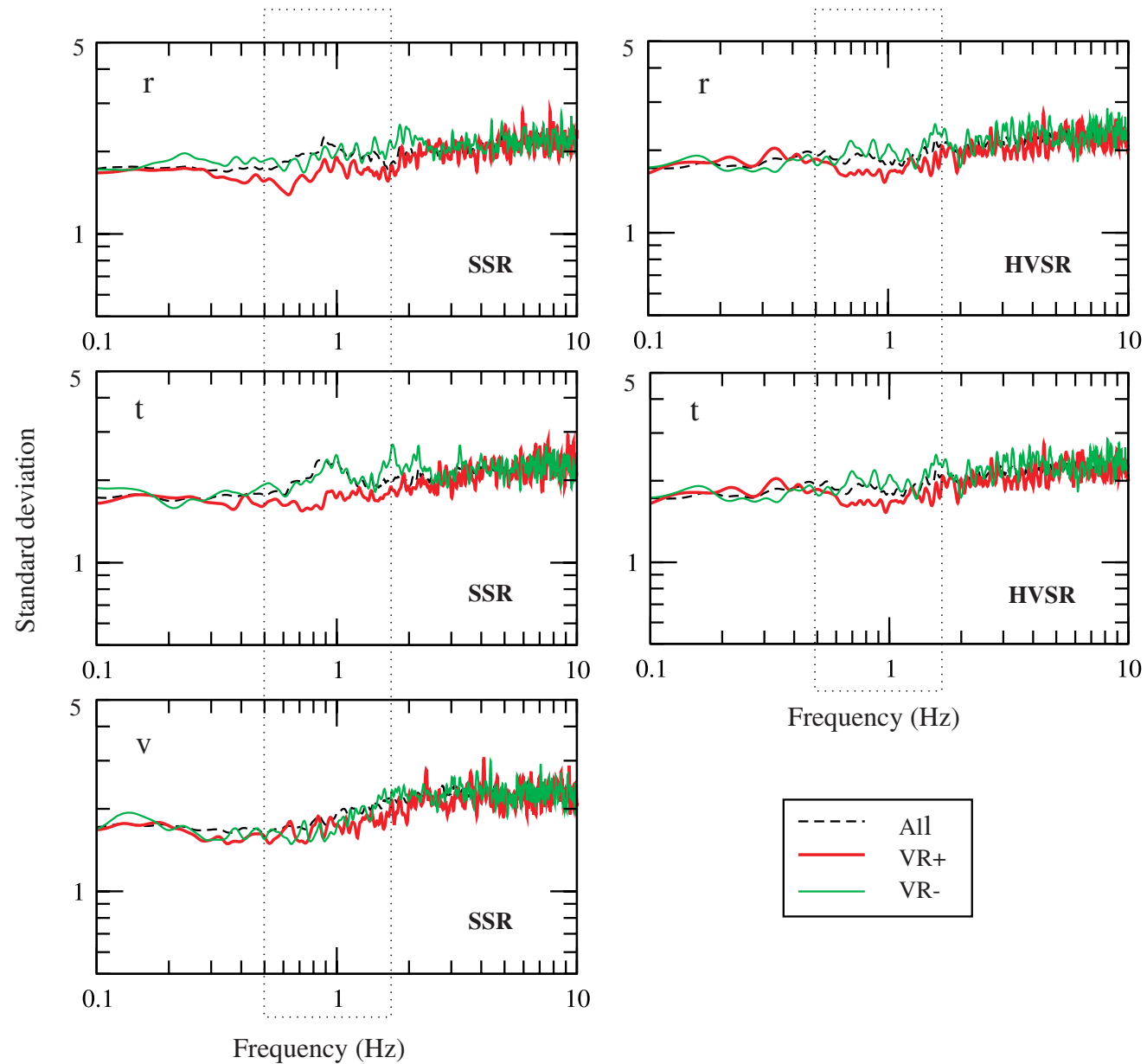

Frequency $(\mathrm{Hz})$

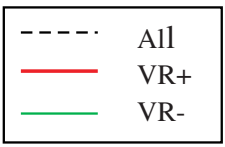

Figure 8. Standard deviation of the mean values of SSR and HVSR for the radial $(r)$, transverse $(t)$, and vertical $(v)$ components. Results are shown for the complete dataset (dashed lines) and for the VR- and VR+ events (thin and thick solid lines respectively). The dotted boxes mark the frequency range $0.5-1.5 \mathrm{~Hz}$. The standard deviation for SSR around the peak for the entire dataset is 2.4 and is almost equal to that of the VR- events, while that for the VR+ events is between 1.5 and 1.8. The color version of this figure is available only in the electronic edition.

frequency range we choose two representative SNR values: the mean and the minimum. The combination of these choices yields four SNR definitions: (1) mean value in the $0.1-10 \mathrm{~Hz}$ frequency range; (2) mean value in the $0.5-1.5 \mathrm{~Hz}$ frequency range; (3) minimum value in the $0.5-1.5 \mathrm{~Hz}$ frequency range; and (4) minimum value in the $0.1-10 \mathrm{~Hz}$ frequency range. We evaluate the impact on spectral ratios of cutting off data according to different values of SNR, using three typical cutoff values, 3,5 , and 10 , which are roughly equidistant on the log scale. Figure 11 illustrates the four definitions of SNR for a random record. For each definition, the thick solid line indicates the value chosen as representative across the frequency range considered, and the thin dashed lines indicate the three cutoff thresholds.

Table 2 (top) shows the percentage of events with respect to the original dataset that is rejected when using the combination of the four SNR definitions and the three cutoff values for each of the three components. It is observed that definition 1 rejects too few events (roughly less than
$10 \%$ of the dataset), while definition 4 rejects too many events (roughly more than 90\%). Definitions 2 and 3 are more useful in filtering the dataset in terms of data volume.

A selection based on SNR for the vertical component is stricter as compared to a selection based on the horizontals. When using definitions 1 and 2, the vertical component rejects up to two times more events than either of the horizontals. When using definitions 3 and 4 , the difference between components is not significant. In practice, the vertical component is less important in site-effects studies than the horizontals if only SSR is used. However, when HVSR is used, it may be necessary to consider also SNR in the vertical component.

Higher cutoff values exclude more events, obviously, but it is for definition 2 that this difference is most pronounced. When raising the threshold from 3 to 5 and then to 10 , the events rejected each time increase twofold (for the horizontal components, this means an increase from $13 \%$ to $28 \%$ and then to $52 \%$ ). We should mention here that the original dataset passed a visual inspection that excluded 

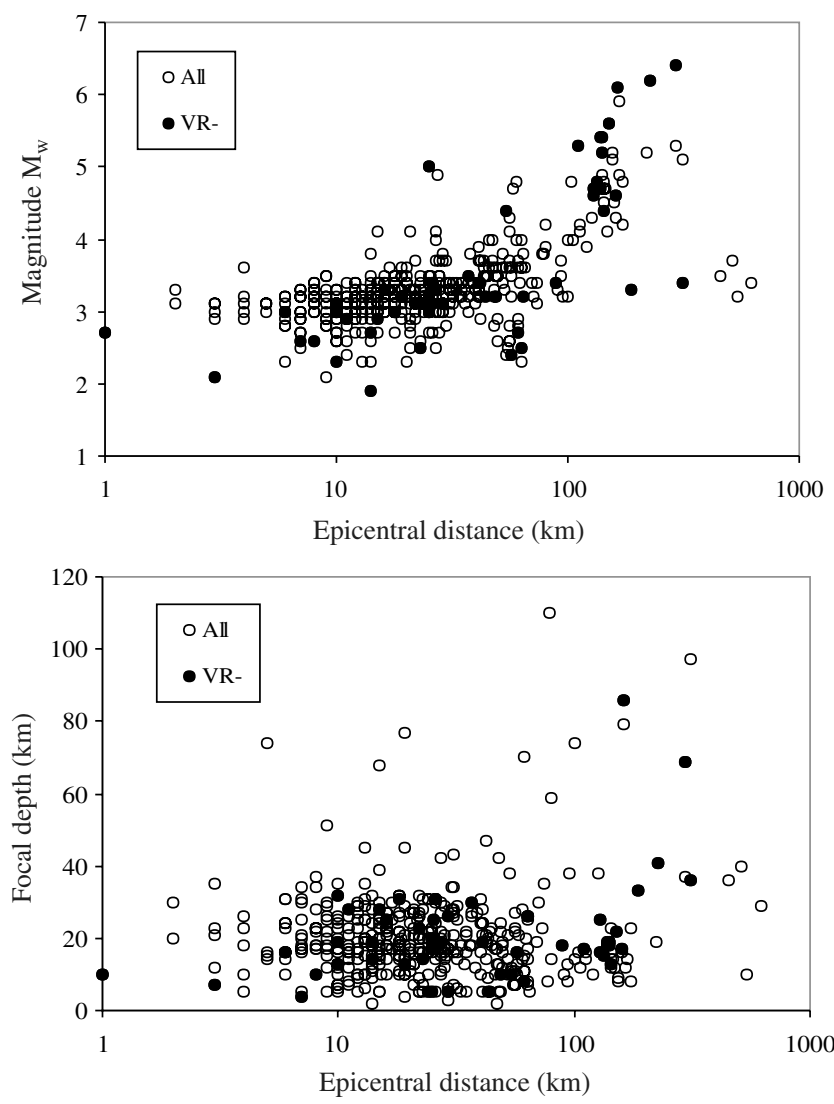

Figure 9. Top diagram: magnitude versus epicentral distance distribution of the complete dataset (open circles) and of the events in the VR- group (solid circles). Bottom diagram: focal depth versus epicentral distance of the complete dataset (open circles) and of the events in the VR- group (solid circles).



Figure 10. Epicentral distribution for the entire dataset (open circles) and for the events in the VR- group (solid circles). The location of CORSSA is marked as a square. The color version of this figure is available only in the electronic edition.

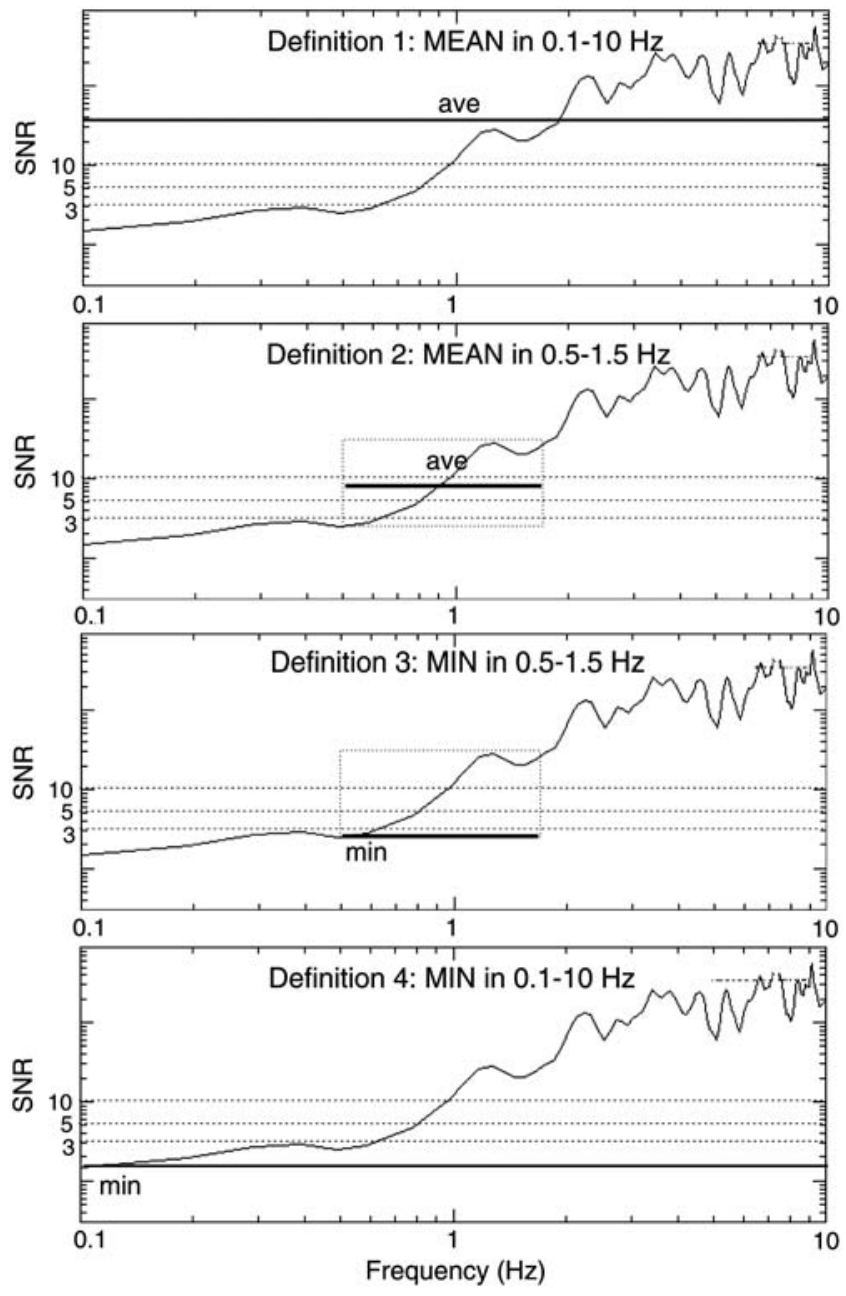

Figure 11. Example of the definitions of SNR, 1 through 4, from top to bottom. In each definition, the graph represents the SNR ( $y$ axis) of a random record, which increases with frequency ( $x$ axis), the solid horizontal line indicates the value chosen as representative in the frequency range considered $(0.5-1.5 \mathrm{~Hz}$ or $0.1-10.0 \mathrm{~Hz}$ ) according to that definition, and the three dashed horizontal lines indicate the cutoff SNR values used (3, 5, and 10).

records of obvious poor quality. Thus, use of a very low SNR cutoff value such as 2 is not effective to screen this dataset, from which events with very low SNR are eliminated from the outset.

\section{Comparison of VR with Signal-to-Noise Ratio}

We can now compare the selection of events obtained using SNR with that obtained using VR. We inspect the events rejected using the SNR cutoff criteria and identify those that belong to the VR- or the VR+ groups. We want to determine whether any of our definitions of SNR systematically rejects the VR- events. For this reason, we introduce the VR - /VR+ ratio, that is, the number of events rejected by a given SNR definition belonging to the VR- group divided by the number of events rejected using SNR and belonging to the VR+ group. Ideally, if the SNR criterion 
Table 2

Percentages of Events (Recorded at the Surface) Rejected by Each SNR Definition*

\begin{tabular}{|c|c|c|c|c|c|}
\hline & \multicolumn{4}{|c|}{ SNR Definition } \\
\hline & & $0.1-10 \mathrm{~Hz}-$ Mean & $0.5-1.5 \mathrm{~Hz}-$ Mean & $0.5-1.5 \mathrm{~Hz}-$ Minimum & $0.1-10 \mathrm{~Hz}-$ Minimum \\
\hline \multicolumn{6}{|c|}{ Percentage of Entire Dataset Rejected for Cutoff Values 3, 5, 10} \\
\hline \multirow[t]{3}{*}{$<3$} & $r$ & $1 \%$ & $13 \%$ & $60 \%$ & $79 \%$ \\
\hline & $t$ & $1 \%$ & $13 \%$ & $62 \%$ & $81 \%$ \\
\hline & $v$ & $4 \%$ & $27 \%$ & $76 \%$ & $87 \%$ \\
\hline \multirow[t]{3}{*}{$<5$} & $r$ & $3 \%$ & $28 \%$ & $74 \%$ & $89 \%$ \\
\hline & $t$ & $3 \%$ & $28 \%$ & $76 \%$ & $87 \%$ \\
\hline & $v$ & $10 \%$ & $45 \%$ & $84 \%$ & $92 \%$ \\
\hline \multirow[t]{3}{*}{$<10$} & $r$ & $10 \%$ & $51 \%$ & $87 \%$ & $95 \%$ \\
\hline & $t$ & $9 \%$ & $52 \%$ & $85 \%$ & $96 \%$ \\
\hline & $v$ & $23 \%$ & $66 \%$ & $91 \%$ & $96 \%$ \\
\hline \multicolumn{6}{|c|}{ Percentage of VR-Group Rejected for Cutoff Values 3, 5, 10} \\
\hline \multirow[t]{3}{*}{$<3$} & $r$ & $0 \%$ & $33 \%$ & $67 \%$ & $75 \%$ \\
\hline & $t$ & $0 \%$ & $29 \%$ & $67 \%$ & $78 \%$ \\
\hline & $v$ & $2 \%$ & $55 \%$ & $71 \%$ & $78 \%$ \\
\hline \multirow[t]{3}{*}{$<5$} & $r$ & $2 \%$ & $53 \%$ & $71 \%$ & $78 \%$ \\
\hline & $t$ & $0 \%$ & $56 \%$ & $73 \%$ & $80 \%$ \\
\hline & $v$ & $11 \%$ & $69 \%$ & $73 \%$ & $84 \%$ \\
\hline \multirow[t]{3}{*}{$<10$} & $r$ & $9 \%$ & $69 \%$ & $75 \%$ & $91 \%$ \\
\hline & $t$ & $7 \%$ & $69 \%$ & $76 \%$ & $87 \%$ \\
\hline & $v$ & $29 \%$ & $71 \%$ & $78 \%$ & $87 \%$ \\
\hline \multicolumn{6}{|c|}{ Ratio of Rejected VR- to Rejected VR+ Events $(\mathrm{VR}-/ \mathrm{VR}+)$} \\
\hline \multirow[t]{3}{*}{$<3$} & $r$ & 0.0 & 18.0 & 3.1 & 1.4 \\
\hline & $t$ & 0.0 & 8.0 & 2.8 & 1.6 \\
\hline & $v$ & 0.5 & 4.3 & 1.8 & 1.3 \\
\hline \multirow[t]{3}{*}{$<5$} & $r$ & 0.3 & 7.3 & 2.1 & 1.2 \\
\hline & $t$ & 0.0 & 10.3 & 1.8 & 1.4 \\
\hline & $v$ & 1.0 & 4.2 & 1.3 & 1.2 \\
\hline \multirow[t]{3}{*}{$<10$} & $r$ & 0.8 & 3.5 & 1.1 & 1.2 \\
\hline & $t$ & 0.7 & 3.2 & 1.3 & 1.1 \\
\hline & $v$ & 1.8 & 2.3 & 1.1 & 1.1 \\
\hline
\end{tabular}

*The numbers in bold indicate the combinations of SNR definitions and cutoff thresholds that make the preferred selection of events.

is successful, we expect large $\mathrm{VR}-/ \mathrm{VR}+$ ratios, indicating that the selection based on SNR managed to disqualify VR- events and keep the VR+ events.

Table 2 (middle) shows the percentage of VR- events, with respect to the VR- group, that is rejected when applying all combinations of the four SNR definitions and the three cutoff values mentioned previously. Definition 1 rejects too few VR- events, while definition 4 rejects most of them. However, we concentrate on the proportion of VR- and $\mathrm{VR}+$ events rejected by each criterion, shown in Table 2 (bottom). Definition 4 does not yield a large contrast between the VR- and VR+ events rejected, meaning it rejects the majority of VR+ events as well. It is definition 2 that maximizes the VR - /VR+ ratio, thus successfully discriminating against the events included in the VR- group.

Consider now the sensitivity of the results to SNR cutoff values. It is for the higher cutoff values (5 and 10) that the majority of VR- events are rejected, but it is for smaller cut- off values ( 3 and 5 ) that the $\mathrm{VR}-/ \mathrm{VR}+$ is higher. We choose the cutoff value of 5 , which yields a VR $-/ \mathrm{VR}+$ ratio around 4 for the vertical component and up to 7 or 10 for the horizontals. In this case there is some difference between the radial and transverse component, so it is suggested that both horizontal components are used in SNR calculations. If HVSR is to be computed, then SNR for the vertical should also be considered.

Our preferred definition for SNR restricts its computation to the frequency range around the fundamental frequency of the site. Thus, it is necessary to have an a priori idea of this frequency. This may be obtained from geotechnical or geophysical data, if available, or from spectral ratios estimated from all available records, before selecting those that will provide the more reliable estimate of site effects. Another alternative is to perform ambient noise measurement, since $H / V$ ratios of noise have long been shown to provide reliable estimates of a site's fundamental frequency. 


\section{Results at Depth}

Signal-to-noise ratio is also calculated for the downhole station at $178 \mathrm{~m}$. The same procedure is followed. The results of the comparison between VR and SNR for the downhole station are given in Table 3, similarly to the results of the surface station. The number of rejected events at depth is larger than those rejected at the surface using the same SNR definitions. As expected, the vertical component does not differ very much from the horizontals at this depth. Other than that, the results are very similar to those of Table 2 , with definition 2 yielding the highest $\mathrm{VR}-/ \mathrm{VR}+$ ratios. Close examination of all events of the dataset shows that the events rejected based on SNR at $178 \mathrm{~m}$ depth include all of those rejected at $0 \mathrm{~m}$. Thus, a selection based on SNR at depth could be considered more conservative. However, VR $-/ \mathrm{VR}+$ ratios for the station at the surface are roughly double those computed for the station at depth, particularly for low cutoff values. The reason is that most of the VR- events rejected based on SNR at depth are also rejected at $0 \mathrm{~m}$, whereas only less than half of the $\mathrm{VR}+$ events rejected based on SNR at $178 \mathrm{~m}$ are rejected at the surface. We find that using an SNR threshold value of 3 at the deep station gives a similar rate of rejected events to that obtained using an SNR threshold of 5 at the surface station.

Signal-to-noise ratio has been reported to improve on firm sites and downhole stations (e.g., Field et al., 1992; Theodulidis et al., 1996). Young et al. (1994) introduced the ratio between the SNR at the deep and shallow levels of a borehole to study the improvement of SNR with depth at a wide frequency range. Douze (1966) showed that SNR improved with depth down to 2 or $3 \mathrm{~km}$; he attributed that to a smaller decrease of the signal amplitude with depth than that of the noise amplitude. Our results show the opposite. Signal-to-noise ratio values at the surface are higher than those at the reference site for all components. Figure 12a shows the ratio of the SNR values between the surface and the deep levels of the borehole (called here SNR0/SNR178) to be

Table 3

Percentages of Events (Recorded at Depth) Rejected by Each SNR Definition*

\begin{tabular}{|c|c|c|c|c|c|}
\hline & & \multicolumn{4}{|c|}{ SNR Definition } \\
\hline & & $0.1-10 \mathrm{~Hz}-$ Mean & $0.5-1.5 \mathrm{~Hz}-$ Mean & $0.5-1.5 \mathrm{~Hz}-$ Minimum & $0.1-10 \mathrm{~Hz}-$ Minimum \\
\hline \multicolumn{6}{|c|}{ Percentage of Entire Dataset Rejected for Cutoff Values 3, 5, 10} \\
\hline \multirow[t]{3}{*}{$<3$} & $r$ & $3 \%$ & $40 \%$ & $82 \%$ & $94 \%$ \\
\hline & $t$ & $5 \%$ & $47 \%$ & $84 \%$ & $95 \%$ \\
\hline & $v$ & $10 \%$ & $55 \%$ & $82 \%$ & $94 \%$ \\
\hline \multirow[t]{3}{*}{$<5$} & $r$ & $12 \%$ & $58 \%$ & $90 \%$ & $96 \%$ \\
\hline & $t$ & $14 \%$ & $64 \%$ & $92 \%$ & $97 \%$ \\
\hline & $v$ & $20 \%$ & $67 \%$ & $89 \%$ & $97 \%$ \\
\hline \multirow[t]{3}{*}{$<10$} & $r$ & $29 \%$ & $77 \%$ & $95 \%$ & $98 \%$ \\
\hline & $t$ & $30 \%$ & $79 \%$ & $96 \%$ & $99 \%$ \\
\hline & $v$ & $32 \%$ & $81 \%$ & $94 \%$ & $99 \%$ \\
\hline \multicolumn{6}{|c|}{ Percentage of VR- Group Rejected for Cutoff Values 3, 5, 10} \\
\hline \multirow[t]{3}{*}{$<3$} & $r$ & $2 \%$ & $65 \%$ & $75 \%$ & $84 \%$ \\
\hline & $t$ & $4 \%$ & $67 \%$ & $73 \%$ & $85 \%$ \\
\hline & $v$ & $11 \%$ & $71 \%$ & $71 \%$ & $84 \%$ \\
\hline \multirow[t]{3}{*}{$<5$} & $r$ & $25 \%$ & $69 \%$ & $78 \%$ & $85 \%$ \\
\hline & $t$ & $27 \%$ & $71 \%$ & $80 \%$ & $87 \%$ \\
\hline & $v$ & $33 \%$ & $71 \%$ & $75 \%$ & $87 \%$ \\
\hline \multirow[t]{3}{*}{$<10$} & $r$ & $45 \%$ & $71 \%$ & $85 \%$ & $91 \%$ \\
\hline & $t$ & $44 \%$ & $73 \%$ & $85 \%$ & $95 \%$ \\
\hline & $v$ & $47 \%$ & $71 \%$ & $78 \%$ & $95 \%$ \\
\hline \multicolumn{6}{|c|}{ Ratio of Rejected VR- to Rejected VR+ Events $(\mathrm{VR}-/ \mathrm{VR}+)$} \\
\hline \multirow[t]{3}{*}{$<3$} & $r$ & 0.5 & 9.0 & 1.5 & 1.1 \\
\hline & $t$ & 0.4 & 6.2 & 1.2 & 1.1 \\
\hline & $v$ & 1.0 & 3.5 & 1.3 & 1.1 \\
\hline \multirow[t]{3}{*}{$<5$} & $r$ & 2.3 & 3.5 & 1.2 & 1.0 \\
\hline & $t$ & 2.1 & 2.6 & 1.1 & 1.1 \\
\hline & $v$ & 2.3 & 2.3 & 1.1 & 1.1 \\
\hline \multirow[t]{3}{*}{$<10$} & $r$ & 2.5 & 1.7 & 1.1 & 1.1 \\
\hline & $t$ & 2.7 & 1.5 & 1.1 & 1.1 \\
\hline & $v$ & 2.4 & 1.4 & 1.0 & 1.1 \\
\hline
\end{tabular}

*The numbers in bold indicate the combinations of SNR definitions and cutoff thresholds that make the preferred selection of events. 
(a)

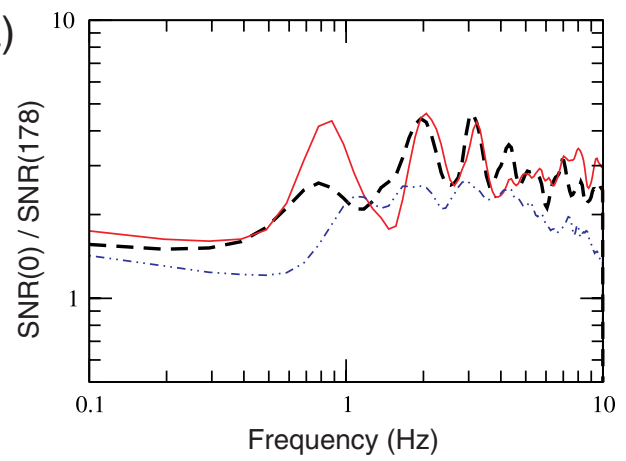

(b)

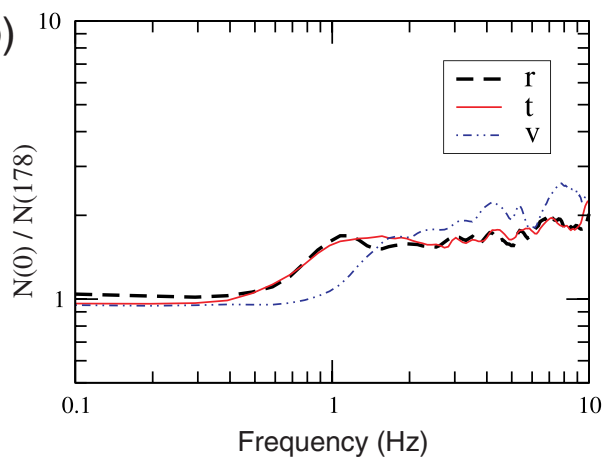

Figure 12. (a) The ratio of SNR between the surface station (0) and the deep station (178) for the three components $(r, t, v)$ as a function of frequency. The values are larger than 1 for all frequencies. (b) Ratio of the noise amplitude between the surface station (0) and the deep station (178) for the three components as a function of frequency. This ratio is smaller than 2 for all frequencies. The color version of this figure is available only in the electronic edition.

larger than 1 at all frequencies. This is in agreement with Douze (1964) who showed that, for shallow depths (down to the first few hundreds of meters) SNR may increase or decrease depending on the site. In the case of CORSSA, signal amplitude decreases faster with depth than noise down to $178 \mathrm{~m}$. This could be due to amplification effects within the soft surface layers; signal amplification at soft sites may partly or fully outweigh that of noise (Bormann, 2002). Figure $12 \mathrm{~b}$ shows the ratio of noise amplitude between the surface and the deep station (called here N0/N178), which is smaller than 2 for all frequencies. This could be related to the sea being very close to the site and generating noise in the borehole. Another explanation is that the reference station is installed only $23 \mathrm{~m}$ into the conglomerate rather than in the deep limestone formation, which is the true bedrock at the site. Scattering from the heterogeneities of the subsoil structure may increase the level of signal-generated noise at $178 \mathrm{~m}$ depth. According to Bormann (2002), the attenuation of noise with depth could require the station to be $100 \mathrm{~m}$ into competent rock.

\section{Conclusions}

A dataset of 473 earthquakes recorded at the surface and at $178 \mathrm{~m}$ depth is used to compute HVSR and SSR at the surface. The resonant frequency of the site has been estimated through various techniques at $0.9 \mathrm{~Hz}$. The standard deviation for both ratios is smaller than 2.0 below the resonance peak and increases up to 2.3 for larger frequencies. Near the resonance peak it is larger for SSR (2.4) than for HVSR (2.0).

We use variance reduction to identify events whose spectral ratios lay closest or farthest from the average, which we consider as the initial best estimate of the transfer function. Variance reduction is computed for a frequency range around the fundamental peak. Two groups of events are formed, including events with the highest (VR+) and lowest (VR-) VR values. The VR- group includes 47 events spectral ratios, and the VR+ group includes 55 . When computing
SSR, the events included in the VR- group systematically miss the fundamental peak. Scatter of the spectral ratios is much smaller for events within the VR+ group than for those within the VR- group. The standard deviation observed for the entire dataset around the SSR peak (up to 2.4) is almost equal to the scatter of the VR-group. The standard deviation using only events in the VR+ group can be lowered to 1.5. Thus, if we remove the VR- events from the dataset, the estimated transfer functions have a smaller uncertainty.

It is not possible to use variance reduction to choose records when computing spectral ratios because usual datasets are small. For this reason, we also analyze SNR. Signal-to-noise ratio is estimated using four definitions. Two different frequency ranges are chosen: around the resonant peak $(0.5-1.5 \mathrm{~Hz})$ and over a wide frequency range (0.1-10.0 Hz). For each range, two representative SNR values are chosen: the mean and the minimum. We test different values for the SNR threshold to eliminate data. These cutoff values are those commonly used in the literature: 3, 5, and 10. Computing SNR over the wide frequency range is found to be inefficient. It is better to use the frequency range around the resonant peak.

Signal-to-noise ratio results are compared with the VR results. When we use SNR computed as the mean over the frequency range $0.5-1.5 \mathrm{~Hz}$ to select events, we effectively reject the events included in the VR- group. When we impose a high SNR threshold (5 or 10), we are able to reject most of the VR- events. However, it is for smaller threshold values (3 and 5) that the rejected events based on SNR have the largest $\mathrm{VR}-/ \mathrm{VR}+$ ratio, rejecting most $\mathrm{VR}-$ events and keeping most VR+ events. A compromise is necessary. We suggest using an SNR threshold value of 5, with SNR computed over the frequency range around the resonant peak. Signal-to-noise ratio can be computed on the horizontal components if HVSR is not of interest, but the vertical component should also be taken into account if HVSR is evaluated. In this case, the vertical component should control the SNR selection criterion. The downside is that we need to have a prior idea of the fundamental frequency of the site. 
Signal-to-noise ratio is also computed for the downhole station at $178 \mathrm{~m}$ depth. At depth, the correlation between VR and SNR in the vertical component does not differ much from that for horizontal components. However, the conclusions regarding SNR at the surface apply also at depth, this time for a threshold cutoff value of 3 .

The procedure we propose could be useful for choosing higher quality earthquake records from available datasets or for roughly assessing the credibility of very small datasets. It involves application of the usual SNR technique, but it is targeted to achieve results comparable to those of a selection of events based on VR. We have shown that the scatter in the estimate of site amplification using a given dataset can be due to particular events and that it is possible to significantly reduce it by removing them. Unfortunately, those events cannot be identified based on magnitude, epicentral distance, source depth, or azimuth between source and recording site. Our results indicate that there may be reason to question SSR derived from very small datasets or at least challenge the certainty with which such results are presented.

\section{Data and Resources}

The accelerograms used in this study were produced by the CORSSA array (available at http://geo.civil.auth.gr/Staff/ dep/pitilakis/CORSSA/; last accessed July 2009), which operates in the framework of an agreement between Aristotle University of Thessaloniki, Greece; National Kapodistrian University of Athens, Greece; and Institut de Radioprotection et de Sûreté Nucléaire, France. Signal processing benefitted significantly from SAC2008 (http://www.iris .edu/software/sac, last accessed August 2010; Goldstein et al., 2003; Goldstein and Snoke, 2005). Some plots were made using Generic Mapping Tools v. 3.4 (www.soest .hawaii.edu/gmt, last accessed August 2010; Wessel and Smith, 1998).

\section{Acknowledgments}

The installation of CORSSA was funded by the European research project CORSEIS (EVG1-1999-00002), while its maintenance continues through the CORSSA agreement. This research was partly conducted during the stay of OJK at Instituto de Ingeniería, Universidad Nacional Autónoma de México (UNAM). OJK thanks Theodoros Chatzigogos and Stella Arnaouti (Aristotle University of Thessaloniki) and Jacopo Selva (Istituto Nazionale di Geofisica e Vulcanologia, Sezione di Bologna) for fruitful discussions. FJCG thanks Coordinación de la Investigación Científica, UNAM, for support. The comments of two anonymous reviewers helped improve the manuscript.

\section{References}

Apostolidis, P., D. Raptakis, K. Pandi, M. Manakou, and K. Pitilakis (2006). Definition of subsoil structure and preliminary ground response in Aigion city (Greece) using microtremor and earthquakes, Soil Dyn. Earthquake Eng. 26, 49-67.

Athanasopoulos, G. A., P. C. Pelekis, and E. A. Leonidou (1999). Effects of surface topography on seismic ground response in the Egion (Greece) 15 June 1995 earthquake, Soil Dyn. Earthquake Eng. 18, 135-149.
Bard, P.-Y. (1998). Microtremor measurements: A tool for site effect estimation?, in The Effects of Surface Geology on Seismic Motion, K. Irikura, K. Kudo, H. Okada, and T. Sasatani (Editors), Balkema, Rotterdam, 1251-1279.

Bonilla, L.-F., J. H. Steidl, J.-C. Gariel, and R. J. Archuleta (2002). Borehole response studies at the Garner Valley Downhole Array, Southern California, Bull. Seismol. Soc. Am. 92, 3165-3179.

Bonilla, L.-F., J. H. Steidl, G. T. Lindley, A. G. Tumarkin, and R. J. Archuleta (1997). Site amplification in the San Fernando Valley, California: Variability of site-effect estimation using the $S$-wave, coda, and $H / V$ methods, Bull. Seismol. Soc. Am. 87, 710-730.

Borcherdt, R. D. (1970). Effects of local geology on ground motion near San Francisco Bay, Bull. Seismol. Soc. Am. 60, 29-61.

Borcherdt, R. D., and J. F. Gibbs (1976). Effects of local geological conditions in the San Francisco Bay region on ground motions and the intensities of the 1906 earthquake, Bull. Seismol. Soc. Am. 66, 467-500.

Bormann, P. (2002). IASPEI New Manual of Seismological Observatory Practice (NMSOP), GeoForschungsZentrum Potsdam, Potsdam, Germany.

Chávez-García, F.-J., G. Pedotti, D. Hatzfeld, and P.-Y. Bard (1990). An experimental study of site effects near Thessaloniki (northern Greece), Bull. Seismol. Soc. Am. 80, 784-806.

Chi, W.-C., D. Dreger, and A. Kaverina (2001). Finite-source modeling of the 1999 Taiwan (Chi-Chi) earthquake derived from a dense strongmotion network, Bull. Seismol. Soc. Am. 91, 1144-1157.

Cramer, C. H. (1995). Weak-motion observations and modeling for the Turkey Flat U.S. site-effects test area near Parkfield, California, Bull. Seismol. Soc. Am. 85, 440-451.

Darragh, R. B., and A. F. Shakal (1991). The site response of two rock and soil station pairs to strong and weak ground motion, Bull. Seismol. Soc. Am. 81, 1885-1899.

Di Giacomo, D., M. R. Gallipoli, M. Mucciarelli, S. Parolai, and S. M. Richwalski (2005). Analysis and modeling of HVSR in the presence of a velocity inversion: The case of Venosa, Italy, Bull. Seismol. Soc. Am. 95, 2364-2372.

Douze, E. J. (1964). Signal and noise in deep wells, Geophysics 29, 721-732.

Douze, E. J. (1966). Noise attenuation in shallow holes, Bull. Seismol. Soc. Am. 56, 619-632.

Field, E., and K. Jacob (1995). A comparison and test of various siteresponse estimation techniques, including three that are not reference-site dependent, Bull. Seismol. Soc. Am. 85, 1127-1143.

Field, E. H., K. H. Jacob, and S. E. Hough (1992). Earthquake site response estimation: A weak-motion case study, Bull. Seismol. Soc. Am. 82, 2283-2307.

Goldstein, P., and A. Snoke (2005). SAC Availability for the IRIS Community, Incorporated Institutions for Seismology Data Management Center Electronic Newsletter. http://www.iris.edu/news/newsletter/ vol7no1/page1.htm (last accessed March 2010).

Goldstein, P., D. Dodge, M. Firpo, and L. Minner (2003). SAC2000: Signal processing and analysis tools for seismologists and engineers, Invited contribution to The IASPEI International Handbook of Earthquake and Engineering Seismology, W. H. K. Lee, H. Kanamori, P. C. Jennings, and C. Kisslinger (Editors), Academic Press, London.

Jarpe, S. P., C. H. Cramer, B. E. Tucker, and A. F. Shakal (1988). A comparison of observations of ground response to weak and strong ground motion at Coalinga, California, Bull. Seismol. Soc. Am. 78, 421-435.

Kato, K., K. Aki, and M. Takemura (1995). Site amplification from coda waves: Validation and application to $S$-wave site response, Bull. Seismol. Soc. Am. 85, 467-477.

Ktenidou, O.-J. (2010). Theoretical and instrumental study of site and topographic effects on strong ground motion in Aegion, Ph.D. Thesis, Aristotle University, Thessaloniki, Greece.

Lachet, C., D. Hatzfeld, P.-Y. Bard, N. Theodulidis, C. Papaioannou, and A. Savvaidis (1996). Site effects and microzonation in the city of Thessaloniki (Greece) comparison of different approaches, Bull. Seismol. Soc. Am. 86, 1692-1703. 
Lermo, J., and F.-J. Chávez-García (1993). Site effect evaluation using spectral ratios with only one station, Bull. Seismol. Soc. Am. 83, 1574-1594.

Lozano, L., M. Herraiz, and K. Singh (2009). Site effect study in central Mexico using $H / V$ and SSR techniques: Independence of seismic site effects on source characteristics, Soil Dynam. Earthquake Eng. 29, 504-516.

Malagnini, L., P. Tricarico, A. Rovelli, R. B. Herrmann, S. Opice, G. Biella, and R. de Franco (1996). Explosion, earthquake, and ambient noise recordings in a Pliocene sediment-filled valley: Inferences on seismic response properties by reference- and non-reference-site techniques, Bull. Seismol. Soc. Am. 86, 670-682.

NIST (2001). NIST/SEMATECH e-Handbook of Statistical Methods, National Institute of Standards and Technology, www.itl.nist.gov/ div898/handbook/ (last accessed July 2009).

Pitilakis, K., K. Makropoulos, P. Bernard, F. Lemeiile, H. Lyon-Caen, C. Berge-Thierry, Th. Tika, M. Manakou, D. Diagourtas, D. Raptakis, P. Kallioglou, K. Makra, D. Pitilakis, and F. Bonilla (2004). The Corinth Gulf Soft Soil Array (CORSSA) to study site effects, Compt. Rendus Geosci. 336, 353-365.

Restrepo-Vélez, L. F., and J. J. Bommer (2003). An exploration of the nature of scatter in ground-motion prediction equations and the implications for seismic hazard assessment, J. Earthquake Eng. 7, 171-199.

Riepl, J., P.-Y. Bard, D. Hatzfeld, C. Papaioannou, and S. Nechtschein (1998). Detailed evaluation of site-response estimation methods across and along the sedimentary valley of Volvi (EURO-SEISTEST), Bull. Seismol. Soc. Am. 88, 488-502.

Rogers, A. M., R. D. Borcherdt, P. A. Covington, and D. M. Perkins (1984). A comparative ground response study near Los Angeles using recordings of Nevada nuclear tests and the 1971 San Fernando earthquake, Bull. Seismol. Soc. Am. 74, 1925-1949.

Şafak, E. (1997). Models and methods to characterize site amplification from a pair of records, Earthquake Spectra 13, 97-139.

Satoh, T., H. Kawase, and S. Matsushima (2001). Differences between site characteristics obtained from microtremors, $S$ waves, $P$ waves, and codas, Bull. Seismol. Soc. Am. 91, 313-334.

Sawazaki, K., H. Sato, H. Nakahara, and T. Nishimura (2009). Time-lapse changes of seismic velocity in the shallow ground caused by strong ground motion shock of the 2000 Western-Tottori earthquake, Japan, as revealed from coda deconvolution analysis, Bull. Seismol. Soc. Am. 99, 352-366.

Scherbaum, F., F. Cotton, and P. Smit (2004). On the use of response spectral-reference data for the selection and ranking of groundmotion models for seismic-hazard analysis in regions of moderate seismicity: The case of rock motion, Bull. Seismol. Soc. Am. 94, 2164-2185.

Skarlatoudis, A. A., C. B. Papazachos, B. N. Margaris, N. Theodulidis, Ch. Papaioannou, I. Kalogeras, E. M. Scordilis, and V. Karakostas
(2003). Empirical peak ground-motion predictive relations for shallow earthquakes in Greece, Bull. Seismol. Soc. Am. 93, 2591-2603.

Steidl, J. H., A. G. Tumarkin, and R. J. Archuleta (1996). What is a reference site?, Bull. Seismol. Soc. Am. 86, 1733-1748.

Theodulidis, N., P.-Y. Bard, R. Archuleta, and M. Bouchon (1996). Horizontal-to-vertical spectral ratio and geological conditions: The case of Garner Valley Downhole Array in Southern California, Bull. Seismol. Soc. Am. 86, 306-319.

Thompson, E. M., L. G. Baise, R. E. Kayen, and B. B. Guzina (2009). Impediments to predicting site response: Seismic property estimation and modeling simplifications, Bull. Seismol. Soc. Am. 99, 2927-2949.

Tucker, B. E., and J. L. King (1984). Dependence of sediment-filled valley response on input amplitude and valley properties, Bull. Seismol. Soc. Am. 74, 153-165.

Wessa, P. (2009). Free Statistics Software, Office for Research Development and Education, version 1.1.23-r3, http://www.wessa.net (last accessed July 2009).

Wessel, P., and W. H. F. Smith (1998). New, improved version of the Generic Mapping Tools released, Eos Trans. AGU 79, 579.

Wong, H. L., M. D. Trifunac, and B. Westermo (1977). Effects of surface and subsurface irregularities on the amplitudes of monochromatic waves, Bull. Seismol. Soc. Am. 67, 353-368.

Young, C. J., E. P. Chael, D. A. Zagar, and J. A. Carter (1994). Variations in noise and signal levels in a pair of deep boreholes near Amarillo, Texas, Bull. Seismol. Soc. Am. 84, 1593-1607.

Laboratory of Soil Mechanics, Foundation Engineering

and Geotechnical Earthquake Engineering

Department of Civil Engineering

Aristotle University of Thessaloniki

P. O. Box 424, GR-54124

Thessaloniki, Greece

ktenidou@ civil.auth.gr

kpitilak@civil.auth.gr

(O.-J.K., K.D.P.)

Instituto de Ingeniería, Universidad Nacional Autónoma de México

Ciudad Universitaria

Coyoacán 04510 México D.F., México

paco@pumas.iingen.unam.mx

(F.J.C.G)

Manuscript received 8 February 2010 